\title{
MECHANISMS IN ENDOCRINOLOGY The physiology of neuronostatin
}

\author{
Mads R Andersen', Malte P Suppli', Jonatan I Bagger', Mikkel B Christensen 1,2,3,4®, Gina L C Yosten ${ }^{5}$, \\ Filip K Knop ${ }^{1,3,6,7} \mathbb{D}$ and Asger Lund $\mathbb{1} 1$
}

${ }^{1}$ Center for Clinical Metabolic Research, Department of Medicine, Gentofte Hospital, University of Copenhagen, Hellerup, Denmark, ${ }^{2}$ Department of Clinical Pharmacology, Bispebjerg Hospital, University of Copenhagen, Copenhagen, Denmark, ${ }^{3}$ Department of Clinical Medicine, Faculty of Health and Medical Sciences, University of Copenhagen, Copenhagen, Denmark, ${ }^{4}$ Copenhagen Center for Translational Research, Bispebjerg Hospital, University of Copenhagen, Copenhagen, Denmark, ${ }^{5}$ Department of Pharmacology and Physiology, Saint Louis University, St. Louis, Missouri, USA, ${ }^{6}$ Steno Diabetes Center Copenhagen, Gentofte, Denmark, and ${ }^{7}$ Novo Nordisk Foundation Center for Basic Metabolic Research, Faculty of Health and Medical Sciences, University of Copenhagen, Copenhagen, Denmark

Correspondence should be addressed to A Lund Email asger.lund.01@regionh.dk

\begin{abstract}
In 2008, the first evidence of a new hormone called neuronostatin was published. The hormone was discovered using a bioinformatic method and found to originate from the same preprohormone as somatostatin. This small peptide hormone of 13 amino acids and a C-terminal amidation was soon found to exert pleiotropic physiological effects. In animal studies, neuronostatin has been shown to reduce food intake and delay gastric emptying and gastrointestinal transit. Furthermore, neuronostatin has been shown to affect glucose metabolism by increasing glucagon secretion during situations when glucose concentrations are low. Additionally, neuronostatin has been shown to affect neural tissue and cardiomyocytes by suppressing cardiac contractility. The effects of neuronostatin have not yet been delineated in humans, but if the effects found in animal studies translate to humans it could position neuronostatin as a promising target in the treatment of obesity, hypertension and diabetes. In this review, we describe the discovery of neuronostatin and the current understanding of its physiological role and potential therapeutic applicability.
\end{abstract}

\section{Introduction}

In 2008, a novel 13-amino acid peptide hormone with a C-terminal amidation originating from the somatostatin preprohormone was discovered and named neuronostatin $(1,2)$. In rodent studies, neuronostatin immunoreactivity was observed in multiple tissues, with the most abundant expression in samples from the pancreas followed by the spleen, stomach, brain stem, hypothalamus, cerebral cortex and cerebellum (2). Neuronostatin was likewise detected in rat plasma, with higher plasma concentrations observed in fasting compared to fed animals (3). Despite

\section{Invited Author's profile}

Asger Lund is a senior specialist registrar in endocrinology at the Department of Medicine and holds a senior research position at the Center for Clinical Metabolic Research at Herlev and Gentofte Hospital, University of Copenhagen, Denmark. His research centres on the pathophysiology of type 2 diabetes, obesity and the regulation of food intake and appetite. He is interested in the integrative role of the gut in human physiology and in particular, the role of glucagon in the pathophysiology

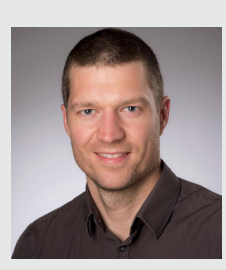
of diabetes.

(C) 2021 European Society of Endocrinology Printed in Great Britain
Published by Bioscientifica Ltd. 
the similarities with somatostatin in both origin and size, it became clear that neuronostatin did not interact with any of the five somatostatin receptors suggesting independent effects of neuronostatin $(2,4)$. Neuronostatin instead was shown to mediate its effects through the previously orphaned G protein-coupled receptor (GPR) 107 located in the spleen, lung, small intestine, stomach, cerebellum, brainstem, hypothalamus, heart, kidney, pancreatic alpha cells and prostate $(5,6)$. The wide distribution of GPR107 indicated that neuronostatin could mediate multiple physiological effects, and subsequent studies in rodents demonstrated that neuronostatin reduced myocardial contractile function, increased blood pressure, affected nociception, increased glucagon secretion from the pancreas during low plasma glucose concentrations, delayed gastric emptying rate and gastrointestinal transit rate and suppressed food and water intake $(3,5,7,8,9,10$, $11,12)$. However, the physiological effects of neuronostatin have not yet been delineated in humans. The objective of this review is to shed light on the current understanding of the physiological role and the therapeutic potential of this newly discovered peptide.

\section{The discovery of neuronostatin}

Neuronostatin was discovered in a collaboration between the US laboratories of Willis KSamson (St. Louis University) and Aaron J W Hsueh (Stanford University) using a novel bioinformatic approach to discover unidentified peptides (2). A search for cleavage sites in already known peptide preprohormones revealed over 200 different potential peptides. The list of novel peptide candidates was reduced by screening for evolutionarily conserved sequences. One of these sequences resided in the preprohormone of somatostatin and would later be named neuronostatin (2). The preprohormone of somatostatin consists of 116 amino acids (Fig. 1) and in this sequence, four novel different isoforms of potentially active peptides consisting of 6, 11, 13 and 19 amino acids, respectively, were predicted based on the presence of conserved basic residues which could serve as potential proteolytic cleavage sites (2). The endogenous peptide was purified from the porcine pancreas and spleen using antibodies raised against a synthetic full-length (19 amino acid) version of the peptide and verified using matrix-assisted laser desorption-ionisation time of flight mass spectrometry verification (2). Two versions of 'neuronostatin' were detected - a 13 and a 19-amino acid version - residing near the N-terminal of the somatostatin pre-prohormone.

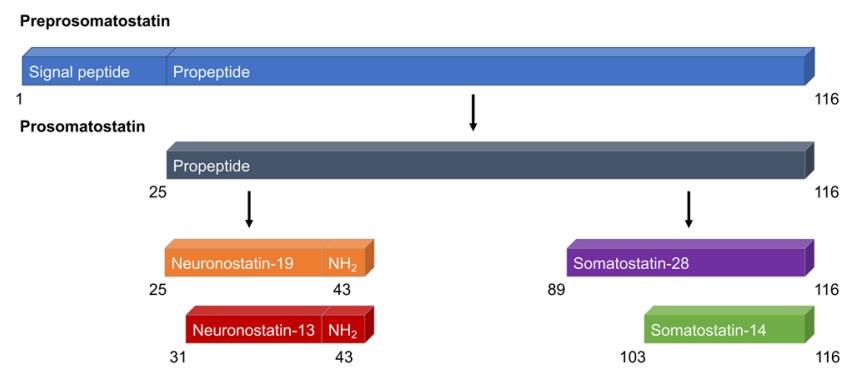

Figure 1

The precursor protein preprosomatostatin and the products derived from it: somatostatin-28, somatostatin-14, neuronostatin-19 and neuronostatin-13. Preprosomatostatin is processed through specific proprotein convertases. The production of somatostatin-14 or somatosatin-28 is tissuespecific and, in some tissues, both versions are produced. There is no evidence of any intermediate products. However, fragments from somatostatin-28 have been observed in tissues only producing somatostatin-14, indicating that it could be processed from somatostatin-28 (43). It is unknown how neuronostatin is processed and degraded. Some of the studies discussed in this review have examined the amidated neuronostatin-19 version.

Because of a glycine residing at the C-terminus, it was predicted to be amidated (2). The 13-amino acid version with a C-terminal amidation (Leu-Arg-Gln-Phe-Leu-GlnLys-Ser-Leu-Ala-Ala-Ala-Ala- $\mathrm{NH}_{2}$ ) was shown to induce the expression of the early response genes c-Fos and c-Jun in a variety of different tissues (2) known as target sites of neuroendocrine and hormonal stimulation (13, 14, 15). Furthermore, the 13 -amino acid version was shown to modulate neural migration, hypothalamic neuron firing and gastric tumour cell proliferation in vitro and had the ability to increase mean arterial pressure and suppress the intake of food and water in rats following intracerebroventricular administration (2). Most studies examining the effects of neuronostatin have utilised the 13-amino acid variant, and in this review, we will focus on this variant; however, a few studies have examined the effects of the 19-amino acid amidated variant, which we will also briefly discuss.

\section{The G protein-coupled receptor 107}

Neuronostatin was shown to mediate its signalling through a protein kinase A (PKA)-dependent mechanism (16). Therefore, Yosten et al. hypothesised that neuronostatin acted through one of the orphan $G$ protein- 
coupled receptors (GPRs) (i.e. GPRs without a known ligand). Tissues known to be responsive to neuronostatin, including the hypothalamus, heart, pancreatic alpha cells and the gastric tumour cell line KATOIII, were screened for the expression of known orphan GPRs. These tissues expressed four orphan receptors in common: GPR56, GPR107, GPR146 and GPR160 (5). The gastric tumour cell line KATOIII was investigated further and treated with siRNA designed to knockdown each of the four receptors (5). After knockdown of the GPR107, neuronostatin no longer elicited stimulatory activity as assessed by c-Fos mRNA expression in the gastric tumour cell line (5), indicating that GPR107 is required for neuronostatinmediated effects in vitro. In addition, neuronostatin increases mean arterial pressure in a biphasic pattern when administered intracerebroventricularly to rats (2, 17), and this effect was abrogated completely after the knockdown of GPR107 in hypothalamus using in vivo siRNA administration (5), providing further evidence that the GPR107 could be neuronostatin's cognate receptor. In another study, performed on pancreatic rat islets and the immortalised mouse alpha cell line $\alpha \mathrm{TC} 1-$ 9, transfection with siRNA targeting GPR107 abrogated neuronostatin-induced increases in PKA phosphorylation and proglucagon mRNA levels (3). Additionally, immunofluorescent analysis of human cadaveric pancreas slices exposed to exogenous neuronostatin revealed co-localisation of neuronostatin and GPR107 in glucagonimmunoreactive cells in islets (3). Ligands of GPRs are known to influence the expression of their receptor (18, 19). Therefore, further evidence for interdependence between neuronostatin and GPR107 was demonstrated by increased GPR107 expression after $6 \mathrm{~h}$ of incubation with neuronostatin in murine pancreatic alpha cells (3). As aforementioned, evidence suggests that the signalling pathway of neuronostatin is mediated via a PKAdependent mechanism, and PKA phosphorylation was shown to increase in pancreatic alpha cells after exposure to neuronostatin (20). Likewise, treatment with two different PKA catalytic subunit inhibitors prevented the effect of neuronostatin on the expression of proglucagon mRNA in mouse alpha cells, further indicating that this effect is PKA-dependent (3). However, PKA activation by neuronostatin was not associated with a rise in the concentration of cyclic AMP (cAMP), suggesting that GPR107-mediated effects of neuronostatin are cAMPindependent (3). Previous studies had demonstrated that a sub-pool of the catalytic subunit of PKA associates with the nuclear factor kappa-light-chain-enhancer of activated B cells (NF-KB) complex $(21,22)$. Therefore, the alpha cells were also treated with an NF-KB inhibitor or an inhibitor of $\mathrm{KB}$ (IKB), respectively, which similarly inhibited the effect of neuronostatin, indicating that neuronostatin activation of the GPR107 is associated with dissociation of a NF-KB-IKB-PKAc complex and subsequent PKA phosphorylation and activity (3). Further evidence of an interaction between neuronostatin and GPR107 was demonstrated in the brain $(23,24)$, pituitary cells (4) and prostate cancer cells (6). Taken together, multiple lines of evidence suggest that GPR107 is the cognate receptor of neuronostatin, but the kinetics of this receptor remain to be fully elucidated.

\section{Physiological effects of neuronostatin in neural tissue}

\section{Overview and interaction of neuronostatin with the central melanocortin system}

Neuronostatin and somatostatin are expressed in a variety of different tissues. In rodent neural tissue, neuronostatin is present in neurons in the hypothalamus, median eminence, hippocampus, motor cortex, amygdala, cerebellum and pituitary gland $(2,4,25,26)$. So far, most studies designed to elucidate the physiological effects of neuronostatin have focussed on tissues in which somatostatin exerts effects (Fig. 2). Somatostatin is known to regulate a wide variety of physiological functions and inhibit the secretion of many hormones including growth hormone release from the pituitary gland. A similar effect of neuronostatin-19 has been found in a study examining cultured baboon pituitary cells where neuronostatin-19 was found to have an inhibitory effect in the basal secretion of growth hormone and luteinising hormone. These findings indicate that neuronostatin-19 might work together with somatostatin to fine-tune growth hormone release (4). However, treatment with neuronostatin-13 showed no effect on growth hormone release from cultured anterior pituitary rat cells (2), which could indicate either a species- or isoform-specific effect of neuronostatin on growth hormone secretion. In cultured rat hypothalamic neurons, neuronostatin and somatostatin were shown to be co-expressed, and somatostatin was shown to decrease calcium influx, whereas neuronostatin was shown to increase intracellular calcium concentrations of neurons (25), indicating that neuronostatin modulates neuronal activity in the hypothalamus in balance with somatostatin. Cell bodies of neuronostatin-positive neurons in rats have been visualised by immunohistochemical staining in the hypothalamic anterior periventricular nucleus 


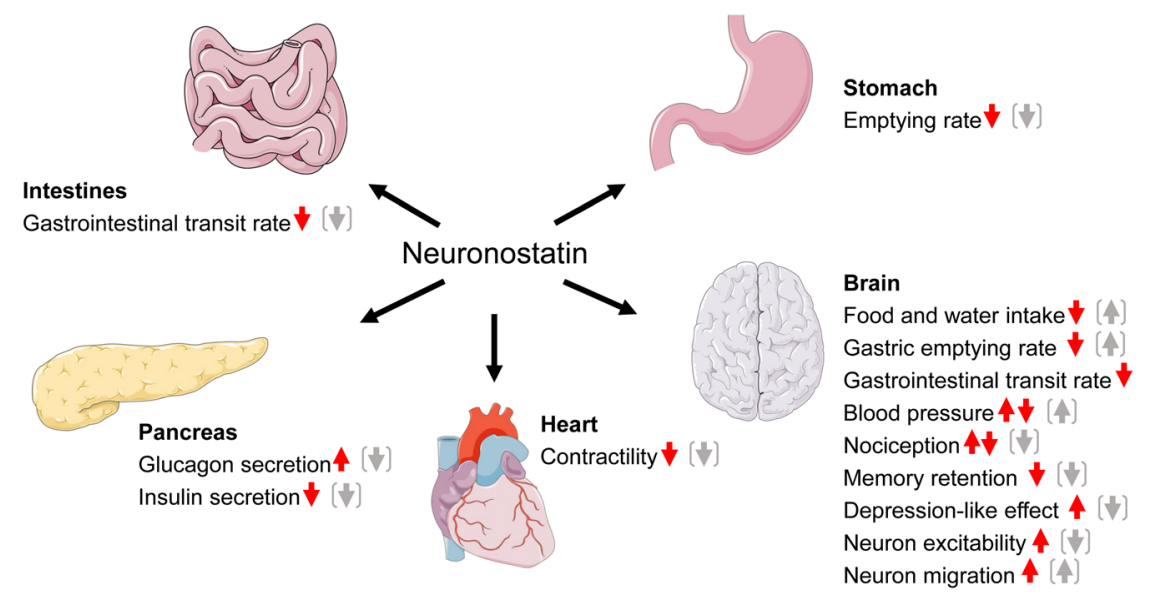

\section{Figure 2}

The physiological effects of neuronostatin as demonstrated in cell and rodent studies. Neuronostatin elicits effects in both neural and peripheral tissues such as the heart, pancreas, intestines and stomach. Arrow up: increase, arrow down: decrease. Grey arrows indicate effects of somatostatin. and the suprachiasmatic nuclei, whereas neuronostatinimmunoreactive axon terminals were observed in the arcuate nucleus and the median eminence (25). Additionally, there are fewer and less densely marked neuronostatin-expressing neurons in the polymorphic layer of the dentate gyrus, the motor cortex, the amygdala and the cerebellum (25). A recent study corroborated these findings by showing neuronostatin-expressing axons in the suprachiasmatic nucleus, the ventromedial hypothalamic nucleus and the arcuate nucleus in rats (26). This study also demonstrated that refeeding rats following a $48 \mathrm{~h}$ fast led to an increase in phosphorylated signal transducer and transcription activator 5 (STAT5) immunoreactivity in neuronostatin-positive neurons (26). In the same study, i.p. injection of two different glutamate receptor agonists increased the expression of c-Fos in neuronostatin-immunoreactive neurons, indicating that the neurotransmitter glutamate might be involved in the peripheral activation of neuronostatin-positive neurons (26). In summary, these findings show that neuronostatin is present in specific neurons in various parts of the brain and gives an insight into which stimuli activate these neurons.

\section{The effects of neuronostatin on food intake}

The periventricular nucleus of the hypothalamus is involved in the regulation of food intake (27). Due to neuronostatin's modulating effect on the excitability in rat hypothalamic paraventricular neurons (2), the effect of neuronostatin on food and water intake was investigated in rats $(2,28,29)$. In these studies, neuronostatin did not exert an effect on food intake upon intrahippocampal administration, but when injected intracerebroventricularly, neuronostatin inhibited food and water intake in a dose-dependent manner $(2,28,29)$.
This effect on food and water intake disappeared when the rats were treated with the melanocortin receptor antagonist SHU9119, suggesting the involvement of the central melanocortin system in neuronostatin-induced inhibition of food intake (29). The effect of peripherally administered neuronostatin (as an i.p. bolus in mice) on food intake has also been examined and was shown to have a dose-dependent inhibitory effect on food intake. It was shown to be significant during the first hour after injection (10), whereas the effect has been demonstrated to last for $4 \mathrm{~h}$ when neuronostatin is administered into the CNS as a bolus (2). Neuropeptides like leptin (30) and nesfatin-1 (31) mediate their appetite-regulating effect through the central oxytocin system, which acts as a downstream regulator of the central melanocortin system (32). However, pre-treatment of rats with the oxytocin antagonist ornithine vasotocin did not prevent the inhibitory effect of neuronostatin on food intake, suggesting that neuronostatin regulates a unique subset of pro-opiomelanocortin neurons that are specifically connected to the melanocortin system (29).

\section{The effects of neuronostatin on gastrointestinal transit}

Neuronostatin has been shown to delay gastrointestinal transit rate (i.e. the ratio between the distance travelled by a test meal (within $30 \mathrm{~min}$ ) and the total length of the intestine) and gastric emptying rate in a dosedependent manner in mice after intracerebroventricular administration (8). These effects were shown to be dependent upon both the central melanocortin system and the opioid system as intracerebroventricular treatment with SHU9119 and naloxone eliminated the effects (8). Additionally, the dose-response curve was demonstrated to be characterised by a bell-shaped form (8). This may be 
a general feature in the mode of action of neuronostatin as discussed later.

\section{The effects of neuronostatin on blood pressure}

The melanocortin system has also been shown to regulate mean arterial pressure (17). Injection of neuronostatin intracerebroventricularly elicits a biphasic rise in mean arterial pressure in rats - initially, sympathetic activity is increased followed by an increase in vasopressin release (17). This increase in vasopressin release is dependent on the central melanocortin system since the hypertensive effect of neuronostatin during the first phase was cancelled after treatment with the central melanocortin receptor antagonist SHU9119 (17).

\section{The effects of neuronostatin on nociception}

Somatostatin is known to modulate nociception and, thus, the effect of neuronostatin on nociception was investigated in mice. Neuronostatin exerted an analgesic effect in the tail immersion test (a thermal test for the evaluation of nociception) (33), and a bell-shaped dose-response curve similar to the aforementioned effects elicited on mean arterial pressure (17) and gastrointestinal transit rate (8). The mice received an intracerebroventricular bolus of between 0.3 and $12 \mathrm{nmol} /$ mouse. The most effective response was observed at $6 \mathrm{nmol} / \mathrm{mouse}$ and the effect declined after administration of $12 \mathrm{nmol} /$ mouse (33). Thus, it seems that the concentration of neuronostatin required to elicit a physiological response needs to be within a certain interval with waning effects at both high and low levels. Intracerebroventricular treatment with the central melanocortin receptor antagonist SHU9119 or naloxone inhibited the analgesic effect of neuronostatin, suggesting that both the central melanocortin system and the opioid system are involved in this effect. Further pharmacologic testing implicated $\mu$-opioid and $x$-opioid receptors in neuronostatin-induced analgesia (33). These findings were challenged by a study showing that intracerebroventricular co-administration of morphine and neuronostatin enhanced the analgesic effects of morphine in a tail immersion test in mice (34). Intracerebroventricular administration of neuronostatin in mice also increased the expression of c-Fos in the periaqueductal grey substance and the nucleus raphe magnus, both of which are important in the regulation of the descending pain pathway (33). The tail immersion test consists of a phasic stimulus of high intensity and is used as a model for acute pain. It has been criticised for not representing clinical pain because of its short duration and absence of tissue damage. Therefore a formalin test consisting of a tonic pain stimulus by intradermal injection of formalin was also conducted (12). Contrary to the tail immersion test, neuronostatin elicited a hyperalgesic response in the formalin test in mice, and this response was similarly shown to be dependent on the central melanocortin and opioid systems (12). Despite contrasting results, these studies provide evidence for the role of neuronostatin in the modulation of acute and tonic pain.

\section{The effects of neuronostatin in learning, memory, and anxiety}

Most of the studies designed to elucidate the neural effects of neuronostatin in rats and mice have been conducted by the administration of the peptide into the ventricles of the brain. Using this method, the whole brain may be affected by the peptide, and therefore, it is not possible to discern in which specific part of the brain the peptide elicits its effect. However, neuronostatin administered directly into the hippocampus of rats has led to an impairment in memory retention both in an object recognition test (i.e. a test to assess time spent exploring novel objects) and in a step-down avoidance test (i.e. a test to train rats in aversive behaviour) (28). Additionally, long-term potentiation generation and thereby the ability to learn and store memories was reduced and anxiolytic effects were observed (28). The hippocampus is a part of the neural system modulating emotion (35), and the role of neuronostatin in the regulation of emotion was investigated. In mice, a forced swim test is typically used to assess the effect of anti-depressive treatments. Neuronostatin was administered intracerebroventricularly into mice and this induced a depression-like effect in the forced swim test, an effect that was abolished when the mice were treated with the central melanocortin receptor antagonist, SHU9119, and a gamma-aminobutyric acid A $\left(\mathrm{GABA}_{\mathrm{A}}\right)$ receptor antagonist (8). In the pathophysiology of Alzheimer's disease, an abnormal deposition of the amyloid peptide $\mathrm{AB} 42$ is observed. This leads to an increase in activation of microglia and astrocytes which in turn leads to damage in neural tissue. To mimic the pathophysiology of Alzheimer's disease, mice were intracerebroventricularly administered with $\mathrm{AB} 42$ and co-administered with neuronostatin. Neuronostatin worsened the detrimental effects of $\mathrm{AB} 42$ on spatial learning and memory (24). The study also demonstrated the involved intracellular pathways as neuronostatin 
increased the concentration of cAMP and expression of GPR107 in the brain and increased the phosphorylation of protein kinase A and glycogen synthase kinase 3 beta while reducing hexokinase activity and levels of ATP in the cortex (24). Recently, the effects of neuronostatin and $\mathrm{AB} 42$ on glucose metabolism in the hippocampus and cerebral cortex were investigated in mice. Neuronostatin was shown to promote the dysfunction elicited by $\mathrm{AB} 42$ in brain glucose metabolism by reducing the expression of mRNA and enzymes responsible for the production of ATP (23), which could indicate a role of neuronostatin in the pathogenesis of Alzheimer's disease.

\section{Summary of central effects}

Taken together, neuronostatin seems to have important effects on neural activation in the hypothalamus, food and water intake, blood pressure, memory, emotion and nociception and it may play a role in the development of Alzheimer's disease. Although neuronostatin is expressed in the same brain areas as somatostatin, it seems to have unique features, which, if translatable to humans, would be interesting to exploit further in relation to neural diseases, including central acromegaly (e.g. growth hormone-secreting tumours), obesity, depression, anxiety, pain and Alzheimer's disease.

\section{Physiological effects in peripheral tissue}

\section{The effects of neuronostatin in the gastrointestinal system}

To investigate the peripheral effects of neuronostatin on the gastrointestinal system, stomachs from mice were treated in vitro with neuronostatin, which showed a concentration-dependent inhibition of gastric tonus, indicating a direct effect of neuronostatin in the stomach of mice (10). Nevertheless, it was unclear whether the effect on gastric emptying was a result of direct action on the smooth muscle tissue or if it was elicited by an indirect action in the enteric neural plexus. Application of the neural voltage-dependent sodium channel blocker, tetrodotoxin, completely abolished the effect of neuronostatin on gastric emptying indicating that the effect might be a result of depolarisation of gastric enteric neurons (10). The same study analysed the gastric emptying rate and gastrointestinal transit rate in mice after injection of an i.p. bolus with neuronostatin and found the gastric emptying rate to be significantly reduced by $\sim 30 \%$ and gastrointestinal transit rate to be significantly decreased by $\sim 10 \%$ (10). In comparison, central administration of neuronostatin in mice similarly reduced gastric emptying rate by $\sim 14 \%$ and gastrointestinal transit by $\sim 22 \%$ (8). These findings suggest that in rodents, neuronostatin reduces gastric emptying and gastrointestinal transit rate through both central and peripheral pathways.

\section{Neuronostatin's effect in the pancreas}

In the pancreas, neuronostatin is produced in the delta cells (2) and is believed to act via the GPR107 expressed by pancreatic alpha cells (3). An increase in the expression of the early response gene c-Jun was observed in pancreatic islets consistent with an activation of pancreatic alpha cells following i.p. neuronostatin treatment in mice, leading the authors of this study to hypothesise that neuronostatin could influence pancreatic endocrine hormone secretion (2). In 2015, Elrick et al. were the first to visualise GPR107 immunoreactivity in glucagon-expressing alpha cells in human pancreatic tissue by immunohistochemical staining (3). Interestingly, neuronostatin was shown to increase proglucagon mRNA levels and enhance glucagon release under low glucose conditions in primary rat islets and immortalised mouse alpha cells (20); pre-transfection with siRNA inhibiting the expression of GPR107 prevented the induction of proglucagon expression by neuronostatin (3). Additionally, neuronostatin attenuates glucose-stimulated insulin secretion in isolated rat islets, potentially secondarily to a direct effect of neuronostatin on the pancreatic alpha cells (20), although GPR107 mRNA has been detected in human beta cells by RNA sequencing (36). To investigate whether these in vitro effects translated to an in vivo setting, male rats were infused with neuronostatin via a carotid catheter during a glucose challenge (20). This study showed that neuronostatin reduced plasma insulin levels and resulted in a significant delay in glucose clearance. However, the findings were only significant for the first couple of minutes, and the glucose clearance was not significantly affected after $10 \mathrm{~min}$. Also, no effect of neuronostatin on insulin secretion was found when investigated in the immortalised pancreatic beta-cell line INS 832/13 (20). The effect of neuronostatin on pancreatitis has been studied, and pre-treatment with neuronostatin was demonstrated to reduce damaged tissue from taurocholate-induced pancreatitis in rats (37). Overall, the effect of neuronostatin on the endocrine pancreas merits further investigation in rodents and in a translational setting in humans. 


\section{The effect of neuronostatin in cardiomyocytes}

Somatostatin has been shown to reduce contractility in cardiomyocytes (38), and therefore, the effects of neuronostatin in cardiomyocytes have been investigated. Isolated mouse cardiomyocytes and Langendorf wholeheart preparations were treated with neuronostatin, resulting in negative chronotropic and ionotropic effects dependent on a PKA-mediated mechanism (16). However, these findings were in part contradicted by another study using rat cardiomyocytes (7). This study found that neuronostatin only had a negative effect on the contractility of cardiomyocytes when the rats were first infused with the positive inotropic hormone endothelin-1, suggesting that neuronostatin inhibits this effect (7). Furthermore, the study did not demonstrate any increase in troponin I, a known target for phosphorylated PKA, which challenges the finding that negative contractility in mouse hearts by neuronostatin is mediated by a PKAdependent mechanism (7). In order to investigate whether some of these effects could be observed in vivo, mice were injected with an i.p. bolus of neuronostatin, which resulted in suppressed cardiac contractility. This effect was explained by reduced phosphorylation of the sarcoplasmic reticulum $\mathrm{Ca}^{2+}$-ATPase (11). Additionally, activation of AMP-activated protein kinase (AMPK) was observed and co-treatment with an AMPK inhibitor blocked the inhibitory effect on contractility elicited by neuronostatin (11). Altogether, the in vitro and in vivo studies yielded conflicting results, and thus it will be of importance to establish the effect of neuronostatin on cardiomyocytes in humans, as negative contractility in cardiomyocytes could potentially be used in the development of antihypertensive and antianginal medications or - using an antagonistic approach - medications for congestive heart failure.

\section{The potential role of neuronostatin in prostate cancer}

Healthy tissue from the prostate and prostate cancer tissue has been shown to contain the five endogenous somatostatin receptors $(39,40)$ and express the somatostatin gene $(41,42)$. Therefore, the expression of the GPR107 was investigated in prostate cancer cells and found to be expressed (6). Transfection with siRNA designed to silence GPR107 inhibited proliferation of androgen-independent prostate cancer cells, possibly via the protein kinase B pathway (6). Activation of the signalling pathway results in phosphorylation of phosphatidylinositol 3-kinase (PI3K).
PI3K phosphorylates lipids on the plasma membrane and generates the second messenger phosphatidylinositol $(3,4,5)$-triphosphate $\left(\mathrm{PIP}_{3}\right)$, which recruits protein kinase $B$ and, when activated, stimulates cell growth, survival, proliferation, migration and angiogenesis. Treatment with neuronostatin-19 reduced phosphorylation of protein kinase B and the expression of the GPR107, indicating that neuronostatin-19 might act similarly to silencing of the GPR107, which could indicate an anti-tumour effect (6).

\section{Summary, perspectives and conclusions}

Since neuronostatin was discovered in 2008, knowledge of its cognate receptor, signalling pathways, expression and physiological effects in different tissues have expanded. The effects elicited by neuronostatin are diverse, affecting both neural and peripheral tissues. When administered intracerebroventricularly neuronostatin has been demonstrated to decrease food and water intake, increase blood pressure, induce depression-like and anxiolytic effects, impair memory retention, affect nociception and decelerate gastric emptying and gastrointestinal transit rates. Administration of neuronostatin in peripheral gastrointestinal tissues also reduces food intake, gastric emptying and gastrointestinal transit rates. Interestingly, neuronostatin seems to evoke a potentiating effect on glucagon secretion in pancreatic tissue during low glucose concentrations. Additionally, experimentally induced pancreatitis was less severe in animals pre-treated with neuronostatin. In cardiac tissue, neuronostatin exerts negative inotropic and chronotropic effects. When neuronostatin-19's effect in prostate cancer tissue was investigated, an anti-tumour effect was observed.

So far, mice and rats have been the preferred animal models in the exploration of the physiological effects of neuronostatin, and whether these effects can be translated into a human setting remains to be elucidated. Nevertheless, if some of these effects translate to humans, they could potentially be utilised in the development of future treatment strategies for a variety of conditions. Noteworthy are the effects seen on gastric emptying and food intake, which may be used for the development of future treatment for overweight or obese individuals. The effects of neuronostatin on cardiomyocytes could potentially result in new antihypertensive treatments. Furthermore, the glucagonotropic effect elicited in the pancreatic alpha cells during low glucose levels is of interest as this effect, if translatable to a human setting, 
could be utilised in the development of new therapeutics designed to counteract insulin-induced hypoglycaemia in patients with insulin-dependent diabetes. Overall, the physiological role of neuronostatin as elucidated from rodent studies is of potential therapeutic interest; however, dedicated studies with neuronostatin in humans are needed to determine the translatability of these findings.

\section{Declaration of interest}

The authors declare that there is no conflict of interest that could be perceived as prejudicing the impartiality of this review. F K Knop is on the editorial board of the European Journal of Endocrinology. He was not involved with the peer-review process of this article.

\section{Funding}

This work did not receive any specific grant from any funding agency in the public, commercial, or not-for-profit sector.

\section{Author contribution statement}

M R A drafted the manuscript. A L conceptualised the review and contributed to the writing of the manuscript. All authors reviewed and edited the manuscript and approved the version of the manuscript to be published.

\section{References}

1 Yosten GLC, Elrick MM, Salvatori A, Stein LM, Kolar GR, Ren J, Corbett JA \& Samson WK. Understanding peptide biology: the discovery and characterization of the novel hormone, neuronostatin. Peptides 201572 192-195. (https://doi.org/10.1016/j. peptides.2015.05.011)

2 Samson WK, Zhang JV, Avsian-Kretchmer O, Cui K, Yosten GLC, Klein C, Lyu RM, Wang YX, Chen XQ, Yang J et al. Neuronostatin encoded by the somatostatin gene regulates neuronal, cardiovascular, and metabolic functions. Journal of Biological Chemistry 2008283 31949-31959. (https://doi.org/10.1074/jbc.M804784200)

3 Elrick MM, Samson WK, Corbett JA, Salvatori AS, Stein LM, Kolar GR, Naatz A \& Yosten GLC. Neuronostatin acts via GPR107 to increase cAMP-independent PKA phosphorylation and proglucagon mRNA accumulation in pancreatic $\alpha$-cells. American Journal of Physiology: Regulatory, Integrative and Comparative Physiology $2016 \mathbf{3 1 0}$ R143-R155. (https://doi.org/10.1152/ajpregu.00369.2014) 4 Luque RM \& Kineman RD. Neuronostatin exerts actions on pituitary that are unique from its sibling peptide somatostatin. Journal of Endocrinology 2018237 217-227. (https://doi.org/10.1530/JOE-18-0135)

5 Yosten GLC, Redlinger LJ \& Samson WK. Evidence for an interaction of neuronostatin with the orphan $\mathrm{G}$ protein-coupled receptor, GPR107. American Journal of Physiology: Regulatory, Integrative and Comparative Physiology 2012303 R941-R949. (https://doi. org/10.1152/ajpregu.00336.2012)

6 Sáez-Martínez P, Jiménez-Vacas JM, León-González AJ, HerreroAguayo V, Montero Hidalgo AJ, Gómez-Gómez E, Sánchez-Sánchez R, Requena-Tapia MJ, Castaño JP, Gahete MD et al. Unleashing the diagnostic, prognostic and therapeutic potential of the neuronostatin/GPR107 system in prostate cancer. Journal of Clinical Medicine 20209 1703. (https://doi.org/10.3390/jcm9061703)

7 Vainio L, Perjes A, Ryti N, Magga J, Alakoski T, Serpi R, Kaikkonen L, Piuhola J, Szokodi I, Ruskoaho H et al. Neuronostatin, a novel peptide encoded by somatostatin gene, regulates cardiac contractile function and cardiomyocyte survival. Journal of Biological Chemistry 2012287 4572-4580. (https://doi.org/10.1074/jbc.M111.289215)

8 Su SF, Yang AM, Yang SB, Wang NB, Lu SS, Wang HH \& Chen Q. Intracerebroventricular administration of neuronostatin delays gastric emptying and gastrointestinal transit in mice. Peptides 2012 35 31-35. (https://doi.org/10.1016/j.peptides.2012.03.013)

9 Yosten GLC. Novel neuropeptides in the control of food intake: neuronostatin and nesfatin-1. Vitamins and Hormones 201392 1-25. (https://doi.org/10.1016/B978-0-12-410473-0.00001-5)

10 Amato A, Baldassano S, Caldara G \& Mulè F. Neuronostatin: peripheral site of action in mouse stomach. Peptides 2015 64 8-13. (https://doi.org/10.1016/j.peptides.2014.12.003)

11 Zhu X, Hu N, Chen X, Zhu MZ, Dong H, Xu X, Luo F, Hua Y, Nair S, Samson WK et al. Neuronostatin attenuates myocardial contractile function through inhibition of sarcoplasmic reticulum Ca2+ATPase in murine heart. Cellular Physiology and Biochemistry 201433 1921-1932. (https://doi.org/10.1159/000362969)

12 Yang SB, Yang AM, Su SF, Wang HH, Wang NB \& Chen Q. Neuronostatin induces hyperalgesia in formalin test in mice. Neuroscience Letters 2012506 126-130. (https://doi.org/10.1016/j. neulet.2011.10.064)

13 Halazonetis TD, Georgopoulos K, Greenberg ME \& Leder P. C-Jun dimerizes with itself and with c-Fos, forming complexes of different DNA binding affinities. Cell 198855 917-924. (https://doi. org/10.1016/0092-8674(88)90147-x)

14 Hoffman GE, Smith MS \& Verbalis JG. c-Fos and related immediate early gene products as markers of activity in neuroendocrine systems. Frontiers in Neuroendocrinology 199314 173-213. (https://doi. org/10.1006/frne.1993.1006)

15 Batterham RL, Cowley MA, Small CJ, Herzog H, Cohen MA, Dakin CL, Wren AM, Brynes AE, Low MJ, Ghatei MA et al. Gut hormone PYY(3-36) physiologically inhibits food intake. Nature 2002 418 650-654. (https://doi.org/10.1038/nature00887)

16 Hua Y, Ma H, Samson WK \& Ren J. Neuronostatin inhibits cardiac contractile function via a protein kinase A- and JNK-dependent mechanism in murine hearts. American Journal of Physiology: Regulatory, Integrative and Comparative Physiology 2009297 R682-R689. (https://doi.org/10.1152/ajpregu.00196.2009)

17 Yosten GLC, Pate AT \& Samson WK. Neuronostatin acts in brain to biphasically increase mean arterial pressure through sympatho-activation followed by vasopressin secretion: the role of melanocortin receptors. American Journal of Physiology: Regulatory, Integrative and Comparative Physiology 2011300 R1194-R1199. (https://doi.org/10.1152/ajpregu.00849.2010)

18 Cai MJ, Dong DJ, Wang Y, Liu PC, Liu W, Wang JX \& Zhao XF G-protein-coupled receptor participates in 20-hydroxyecdysone signaling on the plasma membrane. Cell Communication and Signaling 201412 9. (https://doi.org/10.1186/1478-811X-12-9)

19 May LT, Lin Y, Sexton PM \& Christopoulos A. Regulation of M2 muscarinic acetylcholine receptor expression and signaling by prolonged exposure to allosteric modulators. Journal of Pharmacology and Experimental Therapeutics 2005312 382-390. (https://doi. org/10.1124/jpet.104.073767)

20 Salvatori AS, Elrick MM, Samson WK, Corbett JA \& Yosten GLC. Neuronostatin inhibits glucose-stimulated insulin secretion via direct action on the pancreatic $\alpha$-cell. American Journal of Physiology: Endocrinology and Metabolism 2014306 E1257-E1263. (https://doi. org/10.1152/ajpendo.00599.2013)

21 Dulin NO, Niu J, Browning DD, Ye RD \& Voyno-Yasenetskaya T. Cyclic AMP-independent activation of protein kinase A by vasoactive peptides. Journal of Biological Chemistry $200127620827-20830$. (https://doi.org/10.1074/jbc.C100195200)

22 Sriwai W, Mahavadi S, Al-Shboul O, Grider JR \& Murthy KS. Distinctive $G$ protein-dependent signaling by protease-activated receptor 2 (PAR2) in smooth muscle: feedback inhibition of RhoA 
by cAMP-independent PKA. PLoS ONE 20138 e66743. (https://doi. org/10.1371/journal.pone.0066743)

23 Yang S, Zhou F, Ma M, Yuan Y, Zhao S \& Yu P. Neuronostatin promotion soluble $A \beta 1-42$ oligomers: induced dysfunctional brain glucose metabolism in mice. Neurochemical Research 202045 2474-2486. (https://doi.org/10.1007/s11064-020-03106-y)

24 Yang S, Shao T, Yu P, Cao R, Zhang M, Wen K, Fan M \& He B. Neuronostatin promotes soluble $A \beta 1-42$ oligomers induced spatial learning and memory impairments in mice. Behavioural Brain Research 2019364 62-74. (https://doi.org/10.1016/j.bbr.2019.01.047)

25 Dun SL, Brailoiu GC, Tica AA, Yang J, Chang JK, Brailoiu E \& Dun NJ. Neuronostatin is co-expressed with somatostatin and mobilizes calcium in cultured rat hypothalamic neurons. Neuroscience 2010 166 455-463. (https://doi.org/10.1016/j.neuroscience.2009.12.059)

26 Serter Kocoglu S, Gok Yurtseven D, Cakir C, Minbay Z \& Eyigor O. Glutamatergic activation of neuronostatin neurons in the periventricular nucleus of the hypothalamus. Brain Sciences 202010 217. (https://doi.org/10.3390/brainsci10040217)

27 Berthoud HR. Multiple neural systems controlling food intake and body weight. Neuroscience and Biobehavioral Reviews 200226 393-428. (https://doi.org/10.1016/s0149-7634(02)00014-3)

28 Carlini VP, Ghersi M, Gabach L, Schiöth HB, Pérez MF, Ramirez OA, Fiol de Cuneo M \& de Barioglio SR. Hippocampal effects of neuronostatin on memory, anxiety-like behavior and food intake in rats. Neuroscience 2011197 145-152. (https://doi.org/10.1016/j. neuroscience.2011.09.040)

29 Yosten GLC \& Samson WK. The melanocortins, not oxytocin, mediate the anorexigenic and antidipsogenic effects of neuronostatin. Peptides 201031 1711-1714. (https://doi. org/10.1016/j.peptides.2010.06.017)

30 Blevins JE, Schwartz MW \& Baskin DG. Evidence that paraventricular nucleus oxytocin neurons link hypothalamic leptin action to caudal brain stem nuclei controlling meal size. American Journal of Physiology: Regulatory, Integrative and Comparative Physiology 2004287 R87-R96. (https://doi.org/10.1152/ ajpregu.00604.2003)

31 Maejima Y, Sedbazar U, Suyama S, Kohno D, Onaka T, Takano E, Yoshida N, Koike M, Uchiyama Y, Fujiwara K et al. Nesfatin-1regulated oxytocinergic signaling in the paraventricular nucleus causes anorexia through a leptin-independent melanocortin pathway. Cell Metabolism 200910 355-365. (https://doi. org/10.1016/j.cmet.2009.09.002)

32 Olson BR, Drutarosky MD, Chow MS, Hruby VJ, Stricker EM \& Verbalis JG. Oxytocin and an oxytocin agonist administered centrally decrease food intake in rats. Peptides 199112 113-118. (https://doi. org/10.1016/0196-9781(91)90176-p)
33 Yang A, Ge W, Lu S, Yang S, Su S, Mi Z \& Chen Q. Central administration of neuronostatin induces antinociception in mice. Peptides 201132 1893-1901. (https://doi.org/10.1016/j. peptides.2011.07.010)

34 Yang SB, Yang AM, Shao TJ, Su SF \& Chen Q. Synergistic analgesic effects between neuronostatin and morphine at the supraspinal level. Peptides 201344 105-110. (https://doi.org/10.1016/j. peptides.2013.03.025)

35 Phillips ML, Drevets WC, Rauch SL \& Lane R. Neurobiology of emotion perception I: the neural basis of normal emotion perception. Biological Psychiatry 200354 504-514. (https://doi. org/10.1016/s0006-3223(03)00168-9)

36 Marselli L, Thorne J, Dahiya S, Sgroi DC, Sharma A, Bonner-Weir S, Marchetti P \& Weir GC. Gene expression profiles of beta-cell enriched tissue obtained by laser capture microdissection from subjects with type 2 diabetes. PLOS ONE 20105 e11499. (https://doi. org/10.1371/journal.pone.0011499)

37 Mo J, Yang A, Chen Z, Shao T, Zhang Y \& Chen Q. Neuronostatin ameliorates sodium taurocholate-induced acute pancreatitis in rats. Digestive Diseases and Sciences 201358 2903-2907. (https://doi. org/10.1007/s10620-013-2753-3)

38 Murray F, Bell D, Kelso EJ, Millar BC \& McDermott BJ. Positive and negative contractile effects of somatostatin-14 on rat ventricular cardiomyocytes. Journal of Cardiovascular Pharmacology 200137 324-332. (https://doi.org/10.1097/00005344-200103000-00011)

39 Pedraza-Arévalo S, Hormaechea-Agulla D, Gómez-Gómez E, Requena MJ, Selth LA, Gahete MD, Castaño JP \& Luque RM Somatostatin receptor subtype 1 as a potential diagnostic marker and therapeutic target in prostate cancer. Prostate 201777 1499-1511. (https://doi.org/10.1002/pros.23426)

40 Hormaechea-Agulla D, Jiménez-Vacas JM, Gómez-Gómez E, L-López F, Carrasco-Valiente J, Valero-Rosa J, Moreno MM, SánchezSánchez R, Ortega-Salas R, Gracia-Navarro F et al. The oncogenic role of the spliced somatostatin receptor sst5TMD4 variant in prostate cancer. FASEB Journal 201731 4682-4696. (https://doi.org/10.1096/ fj.201601264RRR)

41 Sinisi AA, Bellastella A \& Pasquali D. Somatostatin and somatostatin receptors in the prostate. Minerva Endocrinologica 200126 159-163.

42 Reubi JC, Waser B, Schaer JC \& Laissue JA. Somatostatin receptor sst1-sst5 expression in normal and neoplastic human tissues using receptor autoradiography with subtype-selective ligands. European Journal of Nuclear Medicine 200128 836-846. (https://doi. org/10.1007/s002590100541)

43 Martinez V. Chapter 180 - Somatostatin. In Handbook of Biologically Active Peptides, 2nd ed., pp. 1320-1329. Ed AJ Kastin. Boston: Academic Press, 2013.

Received 1 April 2021

Revised version received 23 June 2021

Accepted 6 August 2021 